\title{
Application of Food "Kansei" Model to Quality Design for Margarine Conforming the Preference of Consumers
}

\author{
Mario Shibata ${ }^{1}$, Tomohiko Hayashi ${ }^{2}$, Ryuta Konaka $^{2}$, Tetsuya Araki ${ }^{1}$, Yasuyuki Sagara ${ }^{1}$ \\ ${ }^{1}$ Department of Global Agricultural Sciences, Graduate School of Agricultural and Life Sciences, \\ The University of Tokyo, Japan \\ ${ }^{2}$ ADEKA Corporation
}

\section{Introduction}

Food "Kansei" engineering has been playing an increasingly important role, providing innovative stimuli to new food development, product management and marketing strategy in the food industry. Its main object is to develop the consumer-oriented technologies and systems for quantitatively relating the physicochemical and informational attributes of a food with psychological factors regarding palatability as well as eating habits of individual. A Food Kansei Model (FKM) provides a theoretical framework to formulate the causal relationships among the analyzed characteristics, perceived quality and palatability of food products and successfully applied to the design of green tea beverage conforming the preference of consumer (Ikeda et al, 2004) and also process optimization of freeze-drying in designing volatile compounds of coffee (Sagara et al, 2005).

The objective of this study is to develop the methodology for designing table margarine which conform preferences of consumers based on the FKM. To accomplish the objective, appropriate descriptors which are important for consumers were sorted out, and significant perceptual factors and their priority were identified from the data of sensory evaluation which was performed using the proposed descriptors.

\section{Materials and Methods}

Three kinds of table margarine samples were prepared to sort out objectively the appropriate descriptors for sensory evaluation of the table margarine samples, applying both qualitative and statistical approaches. Firstly, 71 descriptors were collected by the brainstorming of 32 consumer and laboratory panels who tasted the 3 samples. Secondly, 45 descriptors were selected through the discussion among 7 consumer panels. Finally, a list of 25 descriptors was sorted out by principal component analysis (PCA), cluster analysis as well as ANOVA, based on the result of sensory evaluation by 62 consumer panels.

Sensory evaluation using the selected 25 descriptors was performed by 81 consumer panels. The panel profiled the appearance, aroma, flavor and texture intensities as well as hedonic rating of the 3 samples employing 7-point descriptive evaluation. To condense the evaluated response into few variables or perceptual factors, the PCA was performed on obtained sensory data.

Using the PC scores and the hedonic ratings, the relationships of perceptual factors and paratability have been analyzed by Multiple regression analysis (MRA). The standard regression coefficients were used to investigate preference for the perceived quality. All calculations were 
carried out applying JMP 6 (SAS Institute Inc.).

\section{Results and Discussion}

The 25 descriptors were summarized by the PCA, and then 8 perceptual factors were identified as "Milky and Sweet (PC \#1)", "Non-fatty (PC \#2)", "Spreadable (PC \#3)", "White (PC \#4)", and "Cheese-like (PC \#5)", "Firm (PC \#6)", "Salty (PC \#7)" and "Diffluentable (PC \#8)".

Standardized regression coefficients $\beta$ obtained by MRA are presented in Table 1, showing the consumer preference. Three perceptual factors, "Milky and Sweet", "Non-fatty", and "Salty", had significant influence on paratability.

The result of MRA indicates that "Non-fatty" was identified as most important factor and "Milky and Sweet" was second that affect pleasantness of table margarine. A significant part of the evaluation of paratability was explained by these factors, and then the factors was applied to the FKM in order to relate the physicochemical properties, perceived qualities, preference and paratability.

The presented methodology would provide effective tools to design the most preferable table margarine, and then would be also applied to other edible oil and fat products.

Table 1. Regression coefficients of hedonic ratings on perceived quality

\begin{tabular}{lcc}
\hline Variable & $\beta$ & $T$ \\
\hline (Constant) & 3.892 & $60.3^{* * *}$ \\
PC \#1 (Milky and Sweet) & 0.362 & $5.6^{* * *}$ \\
PC \#2 (Non-fatty) & 0.686 & $10.6^{* * *}$ \\
PC \#3 (Spreadable) & 0.098 & 1.51 \\
PC \#4 (White) & -0.075 & -1.17 \\
PC \#5 (Cheese-like) & 0.019 & 0.3 \\
PC \#6 (Firm) & 0.012 & 0.19 \\
PC \#7 (Salty) & 0.182 & $2.82 * *$ \\
PC \#8 (Diffluentable) & -0.109 & -1.69 \\
R Squared & 0.407 & \\
R Squared (adjusted) & 0.386 & \\
RMSE & 1.000 & \\
\hline$\beta=$ standardized regression coefficients; & $T=$ T value. ${ }^{* *} p<0.01 ; * * * p<0.001$.
\end{tabular}

\section{Reference}

Ikeda, G., Nagai, H. and Sagara, Y. (2004). Development of Food Kansei Model and Its Application for Designing Tastes and Flavors of Green Tea Beverage. Food Sci. Technol. Res., Vol. 10 No. 4, p. 396.

Sagara, Y., Kaminishi, K., Goto, E., Watanabe, T., Imayoshi, Y. and Iwabuchi, H. (2005). Characteristic Evaluation for Volatile Compounds of Soluble Coffee Depending on Freeze-Drying Conditions. Drying Technol., Vol. 23 No.9-11, p. 2185. 